\section{REVISTA BRASILEIRA DE QUALIDADE DE VIDA}

\title{
Uso de questionários para avaliação da qualidade de vida em Endometriose
}

\section{Use of questionnaires for evaluation of the quality of life in Endometriosis}

\author{
Alessandra Bernadete Trovó de Marqui \\ Universidade Federal do Triângulo Mineiro - UFTM - Uberaba - Brasil \\ alessandratrovo@hotmail.com
}

\section{RESUMO}

OBJETIVO: Identificar quais os questionários utilizados na avaliação da qualidade de vida em mulheres com endometriose.

MÉTODOS: Foi realizada uma pesquisa bibliográfica no Pubmed e Bireme com os seguintes descritores: endometriosis e quality of life em inglês e português, respectivamente. Foram inclusos apenas artigos intimamente relacionados ao tema deste estudo, com abstract/resumo disponível, publicados em língua inglesa ou portuguesa, em seres humanos e cujos descritores estivessem presentes no título do manuscrito e/ou abstract. Foram excluídos aqueles publicados há mais de 10 anos. De acordo com os critérios de inclusão e exclusão, 29 artigos foram selecionados para leitura. RESULTADOS: Foram identificados cinco tipos diferentes de questionários genéricos (EuroQOL5D, Medical Outcomes Study 36 - Item Short Form Health Survey/SF-36, Medical Outcomes Study 12 - Item Short Form Health Survey/SF-12, World Health Organization Quality of Life Assessement-Brief/WHOQOL-bref e 15D) e dois específicos (Endometriosis Health Profile/EHP-30 e Endometriosis Health Profile/EHP-5), que exibem vantagens e desvantagens na aferição da qualidade de vida em pacientes com endometriose. O mais frequente foi o SF-36, em 51,7\% (15/29) e 86,2\% (25/29) dos estudos empregaram apenas instrumentos genéricos. Apenas quatro estudos empregaram instrumentos específicos para mensurar qualidade de vida em mulheres com endometriose.

CONCLUSÕES: O SF-36 prevaleceu como instrumento padrão na análise da qualidade de vida em pacientes com endometriose. As oito escalas individuais deste questionário avaliam os sintomas físicos, emocionais, sociais, psicológicos e funcionais que são extremamente comprometidos nessa condição ginecológica em função dos sintomas clínicos.

PALAVRAS-CHAVE: Endometriose. Qualidade de vida. Questionários.

\section{ABSTRACT}

OBJECTIVE: Identify the questionnaires used in the assessment of quality of life in women with endometriosis.

METHODS: A literature search was performed in Pubmed and Bireme with the following descriptors: endometriosis and quality of life in English and Portuguese, respectively. Articles were included only closely related to the topic of this study, with abstract/summary available, published in English or Portuguese, in humans and which descriptors were present in the title of the 
manuscript and/or abstract. Exclusion criteria were published for over 10 years. According to the inclusion and exclusion criteria, 29 articles were selected for reading.

RESULTS: Five different generic types of questionnaires were identified (EuroQOL-5D, Medical Outcomes Study 36 - Item Short Form Health Survey/SF-36, Medical Outcomes Study 12 - Item Short Form Health Survey/SF-12, World Health Organization Quality of Life AssessementBrief/WHOQOL-bref e 15D) and two specific (Endometriosis Health Profile/EHP-30 e Endometriosis Health Profile/EHP-5), which exhibit advantages and disadvantages in the assessment of quality of life in patients with endometriosis. The most frequent was the SF-36 in $51,7 \%(15 / 29)$ and $86,2 \%(25 / 29)$ of the studies used only generic instruments. Only four studies used specific instruments to measure quality of life in women with endometriosis.

CONCLUSIONS: The SF-36 remained the standard tool for analysis of quality of life in patients with endometriosis. The eight individual scales of this questionnaire assessing physical, emotional, social, psychological and functional symptoms that are extremely committed this gynecological condition in terms of clinical symptoms.

KEYWORDS: Endometriosis. Quality of life. Questionnaires.

\section{Introdução}

A endometriose é uma doença ginecológica benigna, que afeta cerca de 10 a $20 \%$ das mulheres em idade reprodutiva. É caracterizada pela presença de tecido endometrial fora da cavidade uterina. Seus sintomas incluem dor pélvica crônica, dismenorreia, dispareunia, disquezia, disúria e infertilidade (BULUN, 2009). Em relação aos sintomas, um estudo retrospectivo em 892 pacientes com endometriose conduzido por Bellelis et al. (2010) mostrou que 56,8\% referiram dor pélvica crônica, 54,7\% dispareunia de profundidade, 48,3\% alteração intestinal cíclica, 39,8\% infertilidade, $28,4 \%$ dismenorreia incapacitante e $11,7 \%$ alteração urinária cíclica. Além desses sintomas, as pacientes exibiram níveis elevados de ansiedade, depressão (SEPULCRI; AMARAL, 2009) e comprometimento da função sexual (MONTANARI et al., 2013).

O diagnóstico definitivo da endometriose consiste em uma intervenção cirúrgica por videolaparoscopia e uma amostra é obtida para avaliação anatomopatológica, sendo que a classificação é baseada nos critérios estabelecidos pela American Society of Reproductive Medicine (ASRM), que gradua a endometriose em mínima (estádio I), leve (estádio II), moderada (estádio III) ou severa (estádio IV) (ASRM, 1997). As modalidades terapêuticas no tratamento da endometriose têm como foco a melhoria da dor pélvica crônica e da infertilidade (NAVARRO; BARCELOS; SILVA, 2006).

A endometriose é uma doença crônica na qual as pacientes exibem redução na qualidade de vida (QV) (MINSON et al., 2012). Segundo a Organização Mundial de Saúde (OMS), QV é a percepção do indivíduo sobre sua posição na vida, no contexto da cultura e do sistema de valores em que vive e em relação aos seus objetivos, expectativas, padrões e preocupações (FLECK et al., 1999; FLECK et al., 2000). O conceito de QV é multidimensional, subjetivo e mede diferentes aspectos ou domínios da vida, que incluem o bem-estar físico, psíquico, social e funcional (CICONELLI, 2003).

A avaliação da QV é realizada a partir da aplicação de instrumentos que podem ser genéricos (EuroQOL-5D, Medical Outcomes Study 36 - Item Short Form Health Survey/SF-36, SF12, World Health Organization Quality of Life Assessement-Brief/WHOQOL-bref e 15D) ou específicos (Endometriosis Health Profile/EHP-30 e EHP-5).

A análise da QV, por meio da aplicação de questionários de avaliação, permite mensurar o impacto geral de doenças crônicas, como a endometriose, na vida das pacientes. Além disso, poderia ser utilizado como um indicador prognóstico da melhora clínica. Deste modo, essa revisão tem por objetivo identificar os questionários utilizados para avaliação da QV em mulheres com endometriose. 


\section{Métodos}

Foi realizada uma busca eletrônica no PubMed e Bireme/Literatura Latino-Americana e do Caribe em Ciências da Saúde (LILACS) utilizando os seguintes descritores quality of life e endometriosis em inglês e português, respectivamente. A busca nessas bases de dados foi realizada no mês de outubro de 2013.

Foram inclusos apenas artigos intimamente relacionados ao tema deste estudo, com abstract/resumo disponível, publicados em língua inglesa ou portuguesa, em seres humanos e cujos descritores estivessem presentes no título do manuscrito e/ou resumo. Foram excluídos aqueles publicados há mais de 10 anos. Vinte e nove artigos cumpriram os critérios de seleção previamente estabelecidos e foram inclusos nesse estudo.

\section{Resultados}

O Quadro 1 apresenta os 29 estudos que avaliaram QV em mulheres com endometriose e os respectivos instrumentos de avaliação aplicados. Do total, 25 (86,2\%) utilizaram apenas instrumentos genéricos para análise de QV.

Quadro 1 - Lista dos estudos e respectivos questionários empregados para avaliar Qualidade de Vida em mulheres com endometriose

\begin{tabular}{|c|c|c|c|}
\hline Estudo por autor & $\begin{array}{c}\text { Questionários } \\
\text { utilizados }\end{array}$ & Casuística & $\begin{array}{l}\text { País de } \\
\text { origem }\end{array}$ \\
\hline Abbott et al. (2004) & EQ-5D e SF-12 & $\begin{array}{c}39 \text { mulheres com endometriose que se submeteram } \\
\text { a excisão laparoscópica ( } 19=\text { cirurgia atrasada e } \\
20=\text { cirurgia imediata })\end{array}$ & Reino Unido \\
\hline Marques et al. (2004) & SF-36 & 57 mulheres com endometriose e dor pélvica & Brasil \\
\hline Laursen et al. (2005) & SF-36 & $\begin{array}{l}40 \text { mulheres com dor crônica não maligna divididas } \\
\text { em quatro grupos: fibromialgia }(n=10), \\
\text { endometriose }(n=10) \text {, dor lombar }(n=10) \text { e artrite } \\
\text { reumatóide }(n=10) \text { e } 41 \text { mulheres do grupo de } \\
\text { controle saudáveis pareadas por idade. }\end{array}$ & Dinamarca \\
\hline Lyons et al. (2006) & EQ-5D e SF-12 & $\begin{array}{c}\text { Estudo piloto com sete mulheres nulíparas que } \\
\text { sofreram cirurgia laparoscópica coloretal para } \\
\text { endometriose intestinal }\end{array}$ & Austrália \\
\hline $\begin{array}{l}\text { Dubernard et al. } \\
\text { (2006) }\end{array}$ & SF-36 & 52 mulheres com endometriose colorretal & França \\
\hline $\begin{array}{l}\text { Dubernard et al. } \\
\qquad(2008)\end{array}$ & SF-36 & $\begin{array}{c}93 \text { pacientes com endometriose colorretal divididas } \\
\text { em grupo de treinamento }(n=57) \text { e grupo de } \\
\text { validação }(n=36)\end{array}$ & França \\
\hline $\begin{array}{c}\text { Figueiredo e } \\
\text { Nascimento (2008) }\end{array}$ & EHP-30 & 10 mulheres com endometriose & Brasil \\
\hline $\begin{array}{l}\text { Petrelluzzi et al. } \\
\qquad(2008)\end{array}$ & SF-36 & $\begin{array}{l}93 \text { mulheres com endometriose e dor pélvica } \\
\text { crônica e } 82 \text { mulheres saudáveis }\end{array}$ & Brasil \\
\hline $\begin{array}{l}\text { Siedentopf et al. } \\
\text { (2008) }\end{array}$ & SF-36 & $\begin{array}{l}69 \text { pacientes divididas em dois grupos: } 38 \mathrm{com} \\
\text { endometriose e } 31 \text { sem a doença }\end{array}$ & Alemanha \\
\hline $\begin{array}{l}\text { Sepulcri e Amaral } \\
\text { (2009) }\end{array}$ & WHOQOL-bref & 104 mulheres com endometriose pélvica & Brasil \\
\hline Taipale et al. (2009) & $15 \mathrm{D}$ & $\begin{array}{c}337 \text { mulheres que realizaram histerectomia devido à } \\
\text { doença benigna ( } 210 \text { causa uterina ou ovariana, } 20 \\
\text { endometriose, } 51 \text { prolapso uterovaginal e } 56 \\
\text { menorragia) }\end{array}$ & Finlândia \\
\hline Souza et al. (2011) & WHOQOL-bref & $\begin{array}{l}32 \text { mulheres com endometriose e dor pélvica } \\
\text { crônica e } 25 \text { mulheres com outro tipo de dor pélvica }\end{array}$ & Brasil \\
\hline Fourquet et al. (2011) & SF-12 e EHP-5 & 193 mulheres com diagnóstico de endometriose & Porto Rico \\
\hline Nnoaham et al. (2011) & SF-36 & $\begin{array}{c}1418 \text { mulheres provenientes de } 16 \text { centros clínicos } \\
\text { de } 10 \text { países e dessas } 745 \text { tinham diagnóstico de } \\
\text { endometriose }\end{array}$ & $\begin{array}{c}\text { Estudo } \\
\text { multicêntrico }\end{array}$ \\
\hline
\end{tabular}




\begin{tabular}{|c|c|c|c|}
\hline Bassi et al. (2011) & SF-36 & $\begin{array}{c}151 \text { mulheres com endometriose profunda } \\
\text { infiltrante com envolvimento intestinal que } \\
\text { sofreram resecção de um segmento do retosigmóide } \\
\text { por laparoscopia }\end{array}$ & Brasil \\
\hline Mabrouk et al. (2011a) & SF-36 & $\begin{array}{l}100 \text { pacientes com endometriose profunda } \\
\text { infiltrante que realizaram laparoscopia }\end{array}$ & Itália \\
\hline Mabrouk et al. (2011b) & SF-36 & $\begin{array}{c}106 \text { pacientes com endometriose profunda } \\
\text { infiltrante (75 usuárias de contraceptivos orais } \\
\text { combinados e } 31 \text { não receberam terapia hormonal) }\end{array}$ & Itália \\
\hline Tripoli et al. (2011) & WHOQOL-bref & $\begin{array}{c}134 \text { mulheres divididas em três grupos: } \\
\text { Grupo I - } 49 \text { pacientes com dor pélvica crônica } \\
\text { (DPC) e diagnóstico de endometriose; Grupo II - } \\
35 \text { pacientes com DPC e diagnóstico de outra } \\
\text { condição ginecológica; Grupo III - } 50 \text { pacientes } \\
\text { saudáveis } \\
\end{array}$ & Brasil \\
\hline Minson et al. (2012) & SF-36 & 130 mulheres com endometriose & Brasil \\
\hline Simoens et al. (2012) & EQ-5D & $\begin{array}{l}\text { Estudo multicêntrico envolvendo } 909 \text { pacientes } \\
\text { com endometriose de } 10 \text { países }\end{array}$ & $\begin{array}{c}\text { Estudo } \\
\text { multicêntrico }\end{array}$ \\
\hline Zhao et al. (2012) & SF-36 & $\begin{array}{c}87 \text { mulheres com endometriose sob terapia agonista } \\
\text { de hormônio liberador de gonadotrofinas (GnRH) } \\
\text { sendo } 42 \text { no grupo que realizou relaxamento } \\
\text { muscular progressivo e } 45 \text { pacientes no grupo } \\
\text { controle }\end{array}$ & China \\
\hline Setälä et al. (2012) & $15 \mathrm{D}$ & $\begin{array}{c}22 \text { pacientes que realizaram resecção vaginal para } \\
\text { endometriose }\end{array}$ & Finlândia \\
\hline Kössi et al. (2013) & $15 \mathrm{D}$ & $\begin{array}{l}26 \text { pacientes que sofreram ressecção retosigmóide } \\
\text { para endometriose }\end{array}$ & Finlândia \\
\hline Montanari et al. (2013) & SF-36 & $\begin{array}{l}182 \text { mulheres com endometriose profunda } \\
\text { infiltrante }\end{array}$ & Itália \\
\hline Ribeiro et al. (2014) & SF-36 & 40 mulheres com endometriose intestinal profunda & Brasil \\
\hline Vercellini et al. (2013) & EHP-30 & $\begin{array}{c}\text { Grupo que realizou cirurgia }=47 \text { pacientes e Grupo } \\
\text { em uso de progestina }=102 \text { pacientes }\end{array}$ & Itália \\
\hline Zhao et al. (2013) & WHOQOL-bref & $\begin{array}{c}277 \text { pacientes com endometriose divididas em dois } \\
\text { grupos: } 136 \text { sob tratamento de medicina chinesa e } \\
141 \text { sob tratamento de medicina ocidental }\end{array}$ & China \\
\hline $\begin{array}{l}\text { Wickström et al. } \\
\text { (2013) }\end{array}$ & EHP-30 & $\begin{array}{c}42 \text { mulheres com endometriose, } 24 \text { em uso de } \\
\text { lidocaína e } 18 \text { placebo }\end{array}$ & Suécia \\
\hline Strowitzki et al. (2012) & SF-36 & $\begin{array}{l}229 \text { mulheres com endometriose, } 109 \text { tratadas com } \\
\text { dienogest e } 120 \text { com acetato de leuprolide }\end{array}$ & Alemanha \\
\hline
\end{tabular}

Fonte: Autoria própria (2014).

Uma análise mais detalhada do Quadro 1 evidencia achados interessantes. 11 estudos avaliaram QV em endometriose no período de 6 anos (2004-2009) enquanto que, em apenas 3 anos (2011-2013), 18 estudos foram publicados destacando um crescente interesse nessa área. Esse resultado mostra que a temática QV em endometriose tem sido amplamente investigada e evidencia a preocupação dos pesquisadores em se conhecer como, quanto e em que aspectos essa doença compromete a vida das pacientes. Além disso, cerca de um terço dos estudos sobre QV em endometriose foi realizado em mulheres brasileiras (SEPULCRI; AMARAL, 2009; MINSON et al., 2012; MARQUES et al., 2004; FIGUEIREDO; NASCIMENTO, 2008; PETRELLUZZI et al., 2008; SOUZA et al., 2011; BASSI et al., 2011; TRIPOLI et al., 2011; RIBEIRO et al., 2014).

O Quadro 2 mostra a classificação dos instrumentos utilizados na mensuração da QV em endometriose e os respectivos estudos em que aparecem. O SF-36 foi o questionário mais utilizado, sendo empregado em 15 dos 29 estudos $(51,7 \%)$. 
Quadro 2 - Questionários utilizados na avaliação da Qualidade de Vida em mulheres com endometriose, sua classificação e os respectivos estudos em que aparecem

\begin{tabular}{|c|c|c|c|}
\hline Questionários & Tipo & Estudo & $\mathbf{N}$ \\
\hline SF-36 & Genérico & $\begin{array}{l}\text { Marques et al. (2004) } \\
\text { Laursen et al. (2005) } \\
\text { Dubernard et al. (2006) } \\
\text { Dubernard et al. (2008) } \\
\text { Petrelluzzi et al. (2008) } \\
\text { Siedentopf et al. (2008) } \\
\text { Nnoaham et al. (2011) } \\
\text { Bassi et al. (2011) } \\
\text { Mabrouk et al. (2011a) } \\
\text { Mabrouk et al. (2011b) } \\
\text { Minson et al. (2012) } \\
\text { Zhao et al. (2012) } \\
\text { Montanari et al. (2013) } \\
\text { Ribeiro et al. (2014) } \\
\text { Strowitzki et al. (2012) }\end{array}$ & 15 \\
\hline SF-12 & Genérico & $\begin{array}{l}\text { Abbott et al. (2004)* } \\
\text { Lyons et al. }(2006)^{*} \\
\text { Fourquet et al. }(2011)^{*}\end{array}$ & 03 \\
\hline EuroQol-5D & Genérico & $\begin{array}{l}\text { Abbott et al. }(2004)^{*} \\
\text { Lyons et al. }(2006)^{*} \\
\text { Simoens et al. }(2012)\end{array}$ & 03 \\
\hline $15 \mathrm{D}$ & Genérico & $\begin{array}{l}\text { Taipale et al. (2009) } \\
\text { Setälä et al. (2012) } \\
\text { Kössi et al. (2013) }\end{array}$ & 03 \\
\hline WHOQOL-bref & Genérico & $\begin{array}{c}\text { Sepulcri e Amaral (2009) } \\
\text { Souza et al. (2011) } \\
\text { Tripoli et al. (2011) } \\
\text { Zhao et al. (2013) }\end{array}$ & 04 \\
\hline EHP-30 & Específico & $\begin{array}{c}\text { Figueiredo e Nascimento (2008) } \\
\text { Vercelini et al. (2013) } \\
\text { Wickström et al. (2013) }\end{array}$ & 03 \\
\hline EHP-5 & Específico & Fourquet et al. (2011)* & 01 \\
\hline
\end{tabular}

* Esses três estudos empregaram dois instrumentos de avaliação para mensurar QV.

Fonte: Autoria própria (2014).

Os dados apresentados nos Quadros 1 e 2 mostram que três estudos empregaram dois instrumentos para análise da QV sendo que dois usaram dois tipos diferentes de questionários genéricos (ABBOTT et al., 2004; LYONS et al., 2006) e um estudo, um instrumento genérico e um específico (FOURQUET et al., 2011). Em relação aos instrumentos específicos, o EHP-30 e EHP-5 foram utilizados em apenas quatro estudos (FIGUEIREDO; NASCIMENTO, 2008; FOURQUET et al., 2011; VERCELINI et al., 2013; WICKSTRÖM et al., 2013). O EHP-30 foi aplicado isoladamente (FIGUEIREDO; NASCIMENTO, 2008; VERCELINI et al., 2013; WICKSTRÖM et al., 2013) e o EHP-5 associado a um instrumento genérico (FOURQUET et al., 2011).

\section{Discussão}

A análise dos resultados apresentados nos Quadros 1 e 2 mostrou que os instrumentos genéricos empregados foram o SF-36, SF-12, WHOQOL-bref, EuroQOL-5D e 15D. O SF-36 foi o mais frequente, corroborando os dados já publicados na literatura (DANTAS; SAWADA; MALERBO, 2003; MOTA; NICOLATO, 2008; NEELAKANTAN et al., 2004). Esse instrumento de fácil administração e compreensão foi traduzido para a língua portuguesa e validado por Ciconelli et al. (1999) em pacientes com Artrite Reumatoide, uma doença crônica. É formado por 36 itens, englobando oito escalas: capacidade funcional, aspectos físicos, dor, estado geral de saúde, vitalidade, aspectos sociais, aspectos emocionais e saúde mental. Apresenta um escore final de 0 a 100, onde 0 corresponde ao pior estado geral de saúde e 100 ao melhor estado de saúde. Entre as 
oito escalas, três delas (capacidade funcional, aspectos físicos e dor) correspondem ao Sumário de Componente Físico (Physical Component Sumary/PCS) e o Sumário de Componente Mental (Mental Component Sumary/MCS) abrangem as escalas saúde mental, aspectos emocionais e sociais. Duas das escalas (vitalidade e saúde geral) não apresentam correlação com ambos os componentes (WARE JUNIOR, 2000). Uma variação do SF-36 é o SF-12 cujos resultados são expressos como escores de PCS e MCS (WARE JUNIOR; KOSINSKI; KELLER, 1996).

Outro instrumento aplicado em menor escala foi o WHOQOL-bref que corresponde a uma versão abreviada da versão completa - WHOQOL-100 (THE WHOQOL GROUP, 1998a; THE WHOQOL GROUP, 1998b). O WHOQOL-bref avalia quatro domínios de QV: físico, psicológico, relações sociais e meio ambiente. O WHOQOL-100 e WHOQOL-bref foram validados para o português por Fleck et al. (1999) e Fleck et al. (2000), respectivamente, e um maior valor de escore indica melhor QV.

O WHOQOL-bref se fundamenta na concepção de QV da OMS, porquanto os aspectos mensurados abordam a QV global, incluindo os fatores relacionados e não relacionados à saúde. $\mathrm{O}$ SF-36 foi desenvolvido para a mensuração dos aspectos da saúde e atividades geralmente afetadas por condições de saúde, avaliando, então, somente a QV relacionada à saúde (MOTA; NICOLATO, 2008).

O instrumento EuroQol-5D também conhecido apenas como EuroQol ou EQ-5D avalia cinco dimensões da QV: mobilidade, autocuidado, atividades diárias, dor/desconforto e ansiedade/depressão (BROOKS, 1996; RABIN; CHARRO, 2001). Já o instrumento 15D recebe esse nome porque é constituído por 15 questões de múltipla escolha, cada uma representando uma dimensão relacionada com a saúde: movimentação, visão, audição, respiração, sono, alimentação, habilidade verbal, eliminação, atividades diárias, função mental, desconforto e sintomas, depressão, angústia, vitalidade e atividade sexual. Para cada dimensão, o respondente deve escolher um dos cinco níveis que melhor representa seu estado de saúde naquele momento, sendo o melhor nível 1 e o pior 5. O escore 15D geral varia entre 0 e 1 ( 1 representa melhor QV e 0 pior) (HAWTHORNE; RICHARDSON; DAY, 2001; SINTONEN, 2001).

Apenas 4 dos 29 estudos empregaram instrumentos específicos para análise da QV em pacientes com endometriose. O EHP-30 consiste de 30 itens que avaliam 5 dimensões: dor, controle e impotência, bem-estar emocional, apoio social e autoimagem, e de um questionário modular com 23 itens distribuídos em seis escalas: relações sexuais, trabalho, profissão médica, infertilidade, relacionamento com filhos e tratamento. Cada item é avaliado em uma escala de quatro pontos (nunca $=0$, raramente $=1$, às vezes $=2$, muitas vezes $=3$, sempre $=4$ ). As pontuações são transformadas em uma escala de 0 a 100, onde o menor escore significa melhor QV (JONES et al., 2001; JONES; JENKINSON; KENNEDY, 2004a; JONES et al., 2006). Este instrumento foi validado para o português em uma amostra de 54 pacientes com diagnóstico de endometriose (MENGARDA et al., 2008). O EHP-5 é uma versão mais curta do questionário original (EHP-30) e contém 11 questões (JONES; JENKINSON; KENNEDY, 2004b).

Os dados aqui apresentados sugerem o uso do SF-36 como instrumento 'padrão' para análise da QV em pacientes com endometriose. No entanto, uma pesquisa conduzida em 2012 empregou o SF-36 e o EHP-30 para avaliar a QV em 98 mulheres com endometriose da Itália. Os autores concluem que apesar da versão italiana do EHP-30 necessitar de uma revisão estrutural e linguística, este se mostrou mais apropriado que o instrumento genérico (MAIORANA et al., 2012)

Os instrumentos genéricos possibilitam a avaliação simultânea de várias áreas ou domínios e são aplicados a vários tipos de doenças, mas não demonstram alterações em aspectos específicos. Já a abordagem dos instrumentos específicos é restrita aos sintomas e às disfunções específicas de determinado distúrbio, contribuindo pouco para uma visão mais ampla da QV (CICONELLI, 2003; MOTA; NICOLATO, 2008; AGUIAR et al., 2008).

Houve um crescimento exponencial no número de artigos publicados sobre QV e endometriose. Uma pesquisa publicada em 2003 teve por objetivo analisar a produção científica sobre a temática QV produzida pelas universidades públicas do Estado de São Paulo. Dos 53 estudos avaliados, 69,8\% deles foram desenvolvidos em portadores de algum tipo de patologia, 
20,8\% ( $n=11)$ utilizaram instrumentos genéricos e específicos e o SF-36 foi empregado em 18 estudos (34,0\%), sendo utilizado como medida exclusiva em 8 deles e em 10 estudos esteve associado a um instrumento do tipo específico (DANTAS; SAWADA; MALERBO, 2003).

Em relação ao uso isolado ou associado dos instrumentos para avaliação da QV, Aguiar et al. (2008) sugerem o uso combinado do SF-36 e Problem Areas in Diabetes (PAID), ambos já traduzidos e validados para uso no Brasil. O motivo de tal recomendação provavelmente é decorrente do fato de que questionários genéricos e específicos fornecem informações diferentes e exibem particularidades, e, portanto, se associados são complementares.

\section{Considerações finais}

O presente estudo mostra que a QV em mulheres com endometriose têm sido alvo de grande número de pesquisas. O SF-36 prevaleceu como instrumento padrão na análise dessa variável em pacientes com essa condição ginecológica. As oito escalas individuais deste questionário avaliam os sintomas físicos, emocionais, sociais, psicológicos e funcionais que são extremamente comprometidos em endometriose em função dos sintomas clínicos.

\section{Referências}

ABBOTT, J.; HAWE, J.; HUNTER, D.; HOLMES, M.; FINN, P.; GARRY, R. Laparoscopic excision of endometriosis: a randomized, placebo-controlled trial. Fertility and Sterility, v. 82, n. 4, p. 878-884, 2004. rossef

AGUIAR, C. C. T.; VIEIRA, A. P. G. F.; CARVALHO, A. F.; MONTENEGRO-JUNIOR, R. M. Instrumentos de avaliação de qualidade de vida relacionada à saúde no diabetes melito. Arquivos Brasileiros de Endocrinologia \& Metabologia, v. 52, n. 6, p. 931-939, 2008. Crossef

ASRM. Revised American Society for Reproductive Medicine classification of endometriosis: 1996. Fertility and Sterility, v. 67, n. 5, p. 817-821, 1997. rrossef

BASSI, M. A.; PODGAEC, S.; DIAS JUNIOR, J. A.; D'AMICO FILHO, N.; PETTA, C. A.; ABRÃO, M. S. Quality of life after segmental resection of the rectosigmoid by laparoscopy in patients with deep infiltrating endometriosis with bowel involvement. Journal of Minimally Invasive Gynecology, v. 18, n. 6, p. 730-733, 2011. crossef

BELlELIS, P.; DIAS, J. A. JR.; PODGAEC, S.; GONZALES, M.; BARACAT, E. C.; ABRÃO, M. S. Epidemiological and clinical aspects of pelvic endometriosis-a case series. Revista da Associação Médica Brasileira, v. 56, n. 4, p. 461-471, 2010.

BROOKS, R. EuroQol: the current state of play. Health Policy, v. 37, n. 1, p. 53-72, 1996. rrossef

BULUN, S. E. Endometriosis. The New England Journal of Medicine, v. 360, n. 3, p. 268-279, 2009. Crossef

CICONELLI, R. M. Medidas de avaliação de qualidade de vida. Revista Brasileira de Reumatologia, v. 43, n. 2, p. IX-XIII, 2003.

CICONELLI, R. M.; FERRAZ, M. B.; SANTOS, W.; MEINÃO, I.; QUARESMA, M. R. Tradução para a língua portuguesa e validação do questionário genérico de avaliação de qualidade de vida SF36 (Brasil SF-36). Revista Brasileira de Reumatologia, v. 39, n. 3, p. 143-150, 1999. 
DANTAS, R. A. S.; SAWADA, N. O.; MALERBO, M. B. Pesquisas sobre qualidade de vida: revisão da produção científica das universidades públicas do Estado de São Paulo. Revista LatinoAmericana de Enfermagem, v. 11, n. 4, p. 532-538, 2003. Crossef

DUBERNARD, G.; PIKETTY, M.; ROUZIER, R.; HOURY, S.; BAZOT, M.; DARAI, E. Quality of life after laparoscopic colorectal resection for endometriosis. Human Reproduction, v. 21, n. 5, p. 1243-1247, 2006. Crossef

DUBERNARD, G.; ROUZIER, R.; DAVID-MONTEFIORE, E.; BAZOT, M.; DARAI, E. Use of the SF-36 questionnaire to predict quality-of-life improvement after laparoscopic colorectal resection for endometriosis. Human Reproduction, v. 23, n. 4, p. 846-851, 2008. crossef

FIGUEIREDO, J.; NASCIMENTO, R. Avaliação da qualidade de vida de pacientes portadoras de endometriose após inserção do Sistema Intra-Uterino Liberador de Levonorgestrel (SIU-LNg). ACM Arquivos Catarinenses de Medicina, v. 37, n. 4, p. 20-26, 2008.

FLECK, M. P.; LOUZADA, S.; XAVIER, M.; CHACHAMOVICH, E.; VIEIRA, G.; SANTOS, L.; PINZON, V. Application of the Portuguese version of the instrument for the assessment of quality of life of the World Health Organization (WHOQOL-100). Revista de Saúde Pública, v. 33, n. 2, p. 198-205, 1999.

FLECK, M. P.; LOUZADA, S.; XAVIER, M.; CHACHAMOVICH, E.; VIEIRA, G.; SANTOS, L.; PINZON, V. Aplicação da versão em português do instrumento abreviado de avaliação da qualidade de vida "WHOQOL-bref". Revista de Saúde Pública, v. 34, n. 2, p. 178-183, 2000. rossef

FOURQUET, J.; BÁEZ, L.; FIGUEROA, M.; IRIARTE, R. I.; FLORES, I. Quantification of the impact of endometriosis symptoms on health-related quality of life and work productivity. Fertility and Sterility, v. 96, n. 1, p. 107-112, 2011. crossef

HAWTHORNE, G.; RICHARDSON, J.; DAY, N. A. A comparison of the Assessment of Quality of Life (AQoL) with four other generic utility instruments. Annals of Medicine, v. 33, n. 5, p. 358370, 2001. Crossef

JONES, G.; JENKINSON, C.; KENNEDY, S. Development of the Short Form Endometriosis Health Profile Questionnaire: the EHP-5. Quality of Life Research, v. 13, n. 3, p. 695-704, 2004b. crossef

JONES, G.; JENKINSON, C.; KENNEDY, S. Evaluating the responsiveness of the Endometriosis Health Profile Questionnaire: the EHP-30. Quality of Life Research, v. 13, n. 3, p. 705-713, 2004a. rossef

JONES, G.; JENKINSON, C.; TAYLOR, N.; MILLS, A.; KENNEDY, S. Measuring quality of life in women with endometriosis: tests of data quality, score reliability, response rate and scaling assumptions of the Endometriosis Health Profile Questionnaire. Human Reproduction, v. 21, n. 10, p. 2686-2693, 2006. Crossef

JONES, G.; KENNEDY, S.; BARNARD, A.; WONG, J.; JENKINSON, C. Development of an endometriosis quality-of-life instrument: The Endometriosis Health Profile-30. Obstetrics \& Gynecology, v. 98, n. 2, p. 258-264, 2001. Crossef 
KÖSSI, J.; SETÄLÄ, M.; MÄKINEN, J.; HÄRKKI, P.; LUOSTARINEN, M. Quality of life and sexual function 1 year after laparoscopic rectosigmoid resection for endometriosis. Colorectal Disease, v. 15, n. 1, p. 102-108, 2013. Crossef

LAURSEN, B. S.; BAJAJ, P.; OLESEN, A. S.; DELMAR, C.; ARENDT-NIELSEN, L. Health related quality of life and quantitative pain measurement in females with chronic non-malignant pain. European Journal of Pain, v. 9, n. 3, p. 267, 2005. rossef

LIPING, Z; WU, H.; ZHOU, X.; WANG, Q.; ZHU, W.; CHEN, J. Effects of progressive muscular relaxation training on anxiety, depression and quality of life of endometriosis patients under gonadotrophin-releasing hormone agonist therapy. European Journal of Obstetrics \& Gynecology and Reproductive Biology, v. 162, n. 2, p. 211-215, 2012. rossef

LYONS, S. D.; CHEW, S. S. B.; THOMSON, A. J. M.; LENART, M.; CAMARIS, C.; VANCAILLIE, T. G.; ABBOTT, J. A. Clinical and quality-of-life outcomes after fertility-sparing laparoscopic surgery with bowel resection for severe endometriosis. Journal of Minimally Invasive Gynecology, v. 13, n. 5, p. 436-441, 2006. Crossef

MABROUK, M.; FRASCA, C.; GERACI, E.; MONTANARI, G.; FERRINI, G.; RAIMONDO, D.; ALVISI, S.; PARADISI, R.; VILLA, G.; SERACCHIOLI, R. Combined oral contraceptive therapy in women with posterior deep infiltrating endometriosis. Journal of Minimally Invasive Gynecology, v. 18, n. 4, p. 470-474, 2011b. rossef

MABROUK, M.; MONTANARI, G.; GUERRINI, M.; VILLA, G.; SOLFRINI, S.; VICENZI, C.; MIGNEMI, G.; ZANNONI, L.; FRASCA, C.; DI DONATO, N.; FACCHINI, C.; DEL FORNO, S.; GERACI, E.; FERRINI, G.; RAIMONDO, D.; ALVISI, S.; SERACCHIOLI, R. Does laparoscopic management of deep infiltrating endometriosis improve quality of life? A prospective study. Health and Quality of Life Outcomes, v. 9, p. 98, 2011a. Crossef

MAIORANA, A.; SCAFIDI FONTI, G. M.; AUDINO, P.; ROSINI, R.; ALIO, L.; OLIVERI, A. M.; MILITO, A. M. The role of EHP-30 as specific instrument to assess the quality of life of Italian women with endometriosis. Minerva Ginecologica, v. 64, n. 3, p. 231-238, 2012.

MARQUES, A.; BAHAMONDES, L.; ALDRIGHI, J. M.; PETTA, C. A. Quality of life in Brazilian women with endometriosis assessed through a medical outcome questionnaire. The Journal of Reproductive Medicine, v. 49, n. 2, p. 115-120, 2004.

MENGARDA, C. V.; PASSOS, E. P.; PICON, P.; COSTA, A. F.; PICON, P. D. Validação de versão para o português de questionário sobre qualidade de vida para mulher com endometriose (Endometriosis Health Profile Questionnaire - EHP-30). Revista Brasileira de Ginecologia e Obstetrícia, v. 30, n. 8, p. 384-392, 2008. Crossef

MINSON, F. P.; ABRÃO, M. S.; SARDÁ JÚNIOR, J.; KRAYCHETE, D. C.; PODGAEC, S.; ASSIS, F. D. Importance of quality of life assessment in patients with endometriosis. Revista Brasileira de Ginecologia e Obstetrícia, v. 34, n. 1, p. 11-15, 2012.

MONTANARI, G.; DI DONATO, N.; BENFENATI, A.; GIOVANARDI, G.; ZANNONI, L.; VICENZI, C.; SOLFRINI, S.; MIGNEMI, G.; VILLA, G.; MABROUK, M.; SCHIOPPA, C.; VENTUROLI, S.; SERACCHIOLI, R. Women with deep infiltrating endometriosis: sexual satisfaction, desire, orgasm, and pelvic problem interference with sex. The Journal of Sexual Medicine, v. 10, n. 6, p. 1559-1566, 2013. Crossef 
MOTA, J. F.; NICOLATO, R. Qualidade de vida em sobreviventes de acidente vascular cerebral instrumentos de avaliação e seus resultados. Jornal Brasileiro de Psiquiatria, v. 57, n. 2, p. 148156, 2008. rossef

NAVARRO, P. A. A. S.; BARCElOS, I. D. S.; SILVA, J. C. R. Tratamento da endometriose. Revista Brasileira de Ginecologia e Obstetrícia, v. 28, n. 10, p. 612-623, 2006. Crossef

NEELAKANTAN, D.; OMOJOLE, F.; CLARK, T. J.; GUPTA, J. K.; KHAN, K. S. Quality of life instruments in studies of chronic pelvic pain: a systematic review. Journal of Obstetrics \& Gynaecology, v. 24, n. 8, p. 851-858, 2004. Crossef

NNOAHAM, K. E.; HUMMELSHOJ, L.; WEBSTER, P.; D'HOOGHE, T.; DE CICCO NARDONE, F.; DE CICCO NARDONE, C.; JENKINSON, C.; KENNEDY, S. H.; ZONDERVAN, K. T. Impact of endometriosis on quality of life and work productivity: a multicenter study across ten countries. Fertility and Sterility, v. 96, n. 2, p. 366-373, 2011. crossef

PETRELlUZZI, K. F. S.; GARCIA, M. C.; PETTA, C. A.; GRASSI-KASSISSE, D. M.; SPADARI-BRATFISCH, R. C. Salivary cortisol concentrations, stress and quality of life in women with endometriosis and chronic pelvic pain. Stress, v. 11, n. 5, p. 390-397, 2008. Crossef

RABIN, R.; CHARRO, F. EQ-5D: a measure of health status from the EuroQol Group. Annals of Medicine, v. 33, n. 5, p. 337-343, 2001. Crossef

RIBEIRO, P. A. A.; SEKULA, V. G.; ABDALLA-RIBEIRO, H. S.; RODRIGUES, F. C.; AOKI, T.; ALDRIGHI, J. M. Impact of laparoscopic colorectal segment resection on quality of life in women with deep endometriosis: one year follow-up. Quality of Life Research, v. 23, n. 2, p. 639643, 2014. Crossef

SEPULCRI, R. P.; AMARAL, V. F. Depressive symptoms, anxiety, and quality of life in women with pelvic endometriosis. European Journal of Obstetrics \& Gynecology and Reproductive Biology, v. 142, n. 1, p. 53-56, 2009. rossef

SETÄLÄ, M.; HÄRKKI, P.; MATOMÄKI, J.; MÄKINEN, J.; KÖSSI, J. Sexual functioning, quality of life and pelvic pain 12 months after endometriosis surgery including vaginal resection. Acta Obstetricia et Gynecologica Scandinavica, v. 91, n. 6, p. 692-698, 2012. crossef

SIEDENTOPF, F.; TARIVERDIAN, N.; RÜCKE, M.; KENTENICH, H.; ARCK, P. C. Immune status, psychosocial distress and reduced quality of life in infertile patients with endometriosis. American Journal of Reproductive Immunology, v. 60, n. 5, p. 449-461, 2008. Crossef

SIMOENS, S.; DUNSELMAN, G.; DIRKSEN, C.; HUMMELSHOJ, L.; BOKOR, A.; BRANDES, I.; BRODSZKY, V.; CANIS, M.; COLOMBO, G. L.; DELEIRE, T.; FALCONE, T.; GRAHAM, B.; HALIS, G.; HORNE, A.; KANJ, O.; KJER, J. J.; KRISTENSEN, J.; LEBOVIC, D.; MUELLER, M.; VIGANO, P.; WULLSCHLEGER, M.; D'HOOGHE, T. The burden of endometriosis: costs and quality of life of women with endometriosis and treated in referral centres. Human Reproduction, v. 27, n. 5, p. 1292-1299, 2012. crossef

SINTONEN, H. The 15D instrument of health-related quality of life: properties and applications. Annals of Medicine, v. 33, n. 5, p. 328-336, 2001. rossef 
SOUZA, C. A.; OLIVEIRA, L. M.; SCHEFFEL, C.; GENRO, V. K.; ROSA, V.; CHAVES, M. F.; CUNHA FILHO, J. S. Quality of life associated to chronic pelvic pain is independent of endometriosis diagnosis--a cross-sectional survey. Health and Quality of Life Outcomes, v. 9, p. 41, 2011. rossef

STROWITZKI, T.; MARR, J.; GERLINGER, C.; FAUSTMANN, T.; SEITZ, C. Detailed analysis of a randomized, multicenter, comparative trial of dienogest versus leuprolide acetate in endometriosis. International Journal of Gynaecology \& Obstetrics, v. 117, n. 3, p. 228-233, 2012. crossef

TAIPALE, K.; LEMINEN, A.; RÄSÄNEN, P.; HEIKKILÄ, A.; TAPPER, A. M.; SINTONEN, H.; ROINE, R. P. Costs and health-related quality of life effects of hysterectomy in patients with benign uterine disorders. Acta Obstetricia et Gynecologica Scandinavica, v. 88, n. 12, p. 1402-1410, 2009. rossef

TRIPOLI, T. M.; SATO, H.; SARTORI, M. G.; ARAUJO, F. F.; GIRÃO, M. J. B. C.; SCHOR, E. Evaluation of quality of life and sexual satisfaction in women suffering from chronic pelvic pain with or without endometriosis. The Journal of Sexual Medicine, v. 8, n. 2, p. 497-503, 2011. crossef

VERCELLINI, P.; FRATTARUOLO, M. P.; SOMIGLIANA, E.; JONES, G. L.; CONSONNI, D.; ALBERICO, D.; FEDELE, L. Surgical versus low-dose progestin treatment for endometriosisassociated severe deep dyspareunia II: effect on sexual functioning, psychological status and healthrelated quality of life. Human Reproduction, v. 28, n. 5, p. 1221-1230, 2013. rossef

WARE JUNIOR, J. E.; KOSINSKI, M.; KELLER, S. D. A 12-Item Short-Form Health Survey: construction of scales and preliminary tests of reliability and validity. Medical Care, v. 34, n. 3, p. 220-233, 1996. rossef

WARE JUNIOR, J. E. SF-36 health survey update. Spine (Phila Pa 1976), v. 25, n. 24, p. 31303139, 2000.

THE WHOQOL GROUP. Development of the World Health Organization WHOQOL-BREF quality of life assessment. The WHOQOL Group. Psychological Medicine, v. 28, n. 3, p. 551-558, 1998a. Crossef

THE WHOQOL GROUP. The World Health Organization Quality of Life Assessment (WHOQOL): development and general psychometric properties. Social Science \& Medicine, v. 46, n. 12 , p. $1569-1585,1998$ b. Crossef

WICKSTRÖM, K.; BRUSE, C.; SJÖSTEN, A.; SPIRA, J.; EDELSTAM, G. Quality of life in patients with endometriosis and the effect of pertubation with lidocaine - a randomized controlled trial. Acta Obstetricia et Gynecologica Scandinavica, v. 92, n. 12, p. 1375-1382, 2013. Crossef

ZHAO, R. H.; LIU, Y.; TAN, Y.; HAO, Z. P.; MENG, Q. W.; WANG, R.; LONGO, D.; DING, Y. F.; SONG, D. R.; XU, C.; REN, Z. Z.; YANG, Y. H.; WANG, A. M. Chinese medicine improves postoperative quality of life in endometriosis patients: a randomized controlled trial. Chinese Journal of Integrative Medicine, v. 19, n. 1, p. 15-21, 2013. Crossef 